\title{
Recent Progress in CFTR Interactome Mapping and Its Importance for Cystic Fibrosis
}

\author{
Sang Hyun Lim ${ }^{1}$, Elizabeth-Ann Legere ${ }^{2}$, Jamie Snider ${ }^{3}$ and Igor Stagljar ${ }^{1,2,3 *}$ \\ ${ }^{1}$ Department of Biochemistry, University of Toronto, Toronto, ON, Canada, ${ }^{2}$ Department of Molecular Genetics, University of \\ Toronto, Toronto, ON, Canada, ${ }^{3}$ Terrence Donnelly Centre for Cellular and Biomolecular Research, University of Toronto, \\ Toronto, ON, Canada
}

\section{OPEN ACCESS}

Edited by:

Marçal Pastor-Anglada,

University of Barcelona, Spain

Reviewed by:

Csilla Özvegy-Laczka, Institute of Enzymology (MTA), Hungary

Martina Schmidt University of Groningen, Netherlands

Agnieszka Swiatecka-Urban, University of Pittsburgh, United States

*Correspondence: Igor Stagljar igor.stagljar@utoronto.ca

Specialty section:

This article was submitted to Experimental Pharmacology and Drug

Discovery,

a section of the journal

Frontiers in Pharmacology

Received: 15 September 2017 Accepted: 26 December 2017

Published: 17 January 2018

Citation:

Lim SH, Legere E-A, Snider J and Stagljar I (2018) Recent Progress in CFTR Interactome Mapping and Its Importance for Cystic Fibrosis.

Front. Pharmacol. 8:997.

doi: 10.3389/fphar.2017.00997
Cystic Fibrosis Transmembrane Conductance Regulator (CFTR) is a chloride channel found in secretory epithelia with a plethora of known interacting proteins. Mutations in the CFTR gene cause cystic fibrosis (CF), a disease that leads to progressive respiratory illness and other complications of phenotypic variance resulting from perturbations of this protein interaction network. Studying the collection of CFTR interacting proteins and the differences between the interactomes of mutant and wild type CFTR provides insight into the molecular machinery of the disease and highlights possible therapeutic targets. This mini review focuses on functional genomics and proteomics approaches used for systematic, high-throughput identification of CFTR-interacting proteins to provide comprehensive insight into CFTR regulation and function.

Keywords: cystic fibrosis, CFTR interactome, protein-protein interactions, proteomics, interactome mapping

\section{INTRODUCTION}

Cystic Fibrosis (CF) is a life-shortening genetic disease that affects multiple organs including the lungs, pancreas, liver, intestines, and the reproductive tract. The most prevalent symptoms, however, develop in the airways of CF patients with accretion of viscous mucus causing obstruction and an increased susceptibility to bacterial infection. Current treatment of CF is primarily symptom-based with the use of antibiotics to suppress bacterial colonization and physiotherapy to restore mucociliary clearance while enzyme supplements are given to patients who are pancreatic insufficient (Grasemann and Ratjen, 2010; Pasyk et al., 2017). Despite the recent approval of two new drug therapies (KALYDECO $\AA$ and ORKAMBI $\AA$ ), which paved the way for personalized medicine for CF with a possible disease-modifying effect (Fajac and Wainwright, 2017), pulmonary function of CF patients progressively decreases due to recurrent bacterial infection and subsequent inflammation. In addition, the variation in survival and disease severity is extremely large and this heterogeneity is mostly attributed to variation in environmental factors and patients' genetic backgrounds.

CF is caused by mutations in the Cystic Fibrosis Transmembrane Conductance Regulator (CFTR) (Riordan et al., 1989) which is localized primarily in the apical membrane of secretory epithelial cells. The CFTR protein, regulated by phosphorylation and nucleotide binding, functions as an anion channel that mediates the flux of chloride and bicarbonate ions (Riordan, 2008),

Abbreviations: APIP, APAF1 Interacting Protein; CF, Cystic Fibrosis; CFTR, Cystic Fibrosis Transmembrane Conductance Regulator; EHF, ETS Homologous Factor; PPIs, Protein-Protein Interactions; SNP, Single-Nucleotide Polymorphism. 
which provides the driving force for fluid transport. As its name suggests, CFTR also acts as a regulator influencing the activity of a variety of other channels and transporters (Li and Naren, 2010). Disease-causing mutations are associated with the loss of CFTR function at the surface of the airways, which leads to depletion of the airway surface fluid and accumulation of dehydrated and persistent mucus, the hallmark feature of CF (Boucher, 2007). There are currently over 2,000 mutations recorded, however the major mutation (F508del) is present in two-thirds of all CF patients. This mutation causes CFTR misfolding and subsequent retainment in the endoplasmic reticulum where it is targeted for degradation (Cheng et al., 1990). Interestingly, proper folding of the mutant proteins and their function can be partially restored by low temperature rescue (Denning et al., 1992), which reveals that post-translational processes play important roles in the manifestation of CF (Hutt et al., 2010). However, even upon rescue, mutant CFTR displays altered channel activity and reduced protein stability at the cell surface (Okiyoneda et al., 2010; Lukacs and Verkman, 2012), suggesting that there are many interacting proteins involved in proper folding, channel activity, and cell surface stability of CFTR (Riordan, 2005). Although the genetic cause of CF by CFTR mutations has been well studied in the past (Zielenski and Tsui, 1995), considerably less is known about the cellular protein environment of CFTR in CF.

\section{IDENTIFICATION OF CF MODIFIER GENES}

After the realization that the CFTR genotype alone could not account for all the phenotypic variation seen in the disease, linkage analysis and genetic association approaches found evidence of secondary factors affecting CF phenotypes, known as CF modifier genes (Kerem et al., 1990; Slieker et al., 2005; Weiler and Drumm, 2013). Since then, researchers have formed consortium studies and developed genome-wide association studies (GWAS) to elucidate candidate genes in many aspects of CF such as lung function, meconium ileus, and CF related diabetes (Drumm et al., 2005; Wright et al., 2011; Sun et al., 2012; Blackman et al., 2013). As a result, the impact of secondary, non-CFTR genes in modifying the severity of the CF phenotype has gained a growing importance in the field and has been investigated by several groups (Cutting, 2010; Dorfman, 2012). For the most part, the biological roles of these modifier genes in affecting CF phenotypes are not well documented, but, to date, several have been linked to regulation of the inflammatory response ( $\mathrm{Gu}$ et al., 2009a), protein folding and degradation (Emond et al., 2012), apoptosis (Wright et al., 2011), or ion transport (Sun et al., 2012).

One example of a well-known CF gene modifier is EHF (ETS Homologous Factor), involved in the inflammatory response. The regulatory intergenic region of EHF and APIP (APAF1 Interacting Protein) (11p13) was found to contain a singlenucleotide polymorphism (SNP) that had a strong association with lung function in F508del homozygotes (Wright et al., 2011).
This finding was confirmed in three separate studies; these included an additional 2,900 patient genome sequences (Corvol et al., 2015), targeted resequencing analysis (Dang et al., 2016) and a more recent functional follow-up (Stanke et al., 2014). Although less characterized, other SNPS found to be associated with lung function in CF are in areas defined by the genes MUC4, MUC20, HLA Class 11, AGTR2, SLC9A3 (Corvol et al., 2015), SLC26A9, SLC6A14 (Sun et al., 2012), and IFRD1 (Gu et al., 2009a).

Being part of the CFTR genetic interaction network, these novel variants and modifiers help explain why there is a large phenotypic variation in CF patients. In addition, modifier genes may represent possible therapeutic targets for CF treatment in follow-up studies (Cutting, 2010; Yi et al., 2013). However, as interesting as it is to find them, the detailed mechanism by which many of the identified genes affect the severity of CF remains elusive, and whether they physically and/or functionally associate with CFTR itself is still not completely understood (Gu et al., 2009b; Stolzenburg et al., 2017). In order to design improved therapies for treatment of CF, and better understand the mechanism of action by which these therapies work, it is important to obtain a comprehensive knowledge of the proteins associated with both wild type CFTR and the mutated CFTR variants responsible for $\mathrm{CF}$.

\section{IMPORTANCE OF STUDYING CFTR-INTERACTING PROTEINS}

Protein-protein interactions (PPIs) carry out many cellular processes with temporal and spatial precision (Eckford and Bear, 2011). Their inherent dynamic nature allows cells to adjust in response to stimuli and environmental conditions with flexibility in function (Snider et al., 2015). Therefore, dysfunction of PPIs can have systemic consequences resulting from the perturbation of the interconnected cellular networks (Barabási et al., 2011) leading to disease phenotypes such as those seen in CF.

Not surprisingly, CFTR interacts with a wide variety of proteins that play a major role in various aspects of $\mathrm{CF}$ (Riordan, 2005; Li and Naren, 2010). For instance, the phosphorylation and localization of CFTR to the apical membrane, which is crucial for its regulation and proper function, requires a complex PPI network mediated via PDZ interactions (Guggino, 2004; Li and Naren, 2010). CFTR is also known to regulate the activities of other transporters and channels, hence PPIs that can affect the expression or the function of the CFTR channel at the plasma membrane can be of broad physiological significance as well. Furthermore, it has been speculated that cellular background plays an important role in the ability of F508del CFTR to be rescued from degradation (Wang et al., 2008), with targeting of molecular chaperones as a potential therapeutic approach. The importance of PPIs involved in $\mathrm{CF}$ is further strengthened as recent clinical studies that tested CFTR-modulating small molecules have only shown modest effects (Wainwright et al., 2015). 
As a result, there has been a growing interest in CFTR proteomics over the last decade, with analysis of CFTRinteracting proteins becoming an important means of understanding the cell-specific environments associated with wild type and mutant CFTR, and their corresponding functional consequences (Wang and Li, 2001; Li and Naren, 2005; Collawn et al., 2010).

\section{METHODOLOGICAL OVERVIEW}

So far, the approaches used to map protein interactomes can be divided into three classes (Vidal et al., 2011). The first involves combining experimentally validated PPIs from multiple small-scale studies. The second involves computational prediction algorithms, such as the recently developed FpClass (Kotlyar et al., 2014), which make use of a variety of available known datasets and protein features to generate lists of predicted potential interactions. And finally, the third employs systematic high-throughput experimental mapping strategies. Notably, mapping systematic and comprehensive interaction networks was challenging due to low accuracy during early implementations (von Mering et al., 2002). However, advances in data analysis and methods to empirically assess protein interaction mapping quality have enabled robust validation of the accuracy and sensitivity of datasets acquired using high-throughput approaches, something which isn't possible with the results of smallscale experiments used for curated databases (Venkatesan et al., 2009; De Las Rivas and Fontanillo, 2010; Rolland et al., 2014). There are two general types of methodologies widely used for large-scale PPI mapping; variations of two-hybrid/protein complementation systems and protein purification techniques followed by mass spectrometry (De Las Rivas and Fontanillo, 2010). Two-hybrid/protein complementation datasets mostly contain binary interactions while mass spectrometry datasets contain a mix of both direct associations and indirect interactions that may occur in the context of protein complexes.

Elucidation of protein interactors of CFTR using systematic strategies has traditionally been difficult due to the complex biochemical features of this integral membrane protein. Many of the protein purification-based techniques employed are not high throughput-compatible and often require optimization for each protein complex examined. Additional technical constraints such as the low endogenous expression of CFTR and the lack of high-quality anti-CFTR antibodies also present complications and often demand use of epitopetagged CFTR. Despite these difficulties, assays such as GST-pull downs, co-immunoprecipitation, and affinity purification-mass spectrometry (AP-MS) have been used to elucidate potential CFTR-interacting proteins (Sun et al., 2000a; Cheng et al., 2002; Scroggins et al., 2007). Genetic methods such as the yeast two hybrid have also been used, albeit with just the cytosolic domains of CFTR, to identify interactors (Raghuram et al., 2002; Kim et al., 2004; Duan et al., 2012). However, our knowledge of the comprehensive human CFTR interactome is still at an early stage, as only a few studies have examined CFTR interactions on a global scale (Wang et al., 2006; Pankow et al., 2016).

\section{CURRENT KNOWLEDGE OF THE GLOBAL CFTR INTERACTOME}

CFTR-interacting proteins identified to date can be broadly categorized based on the cellular location where the interaction takes place (Figure 1). These include the nucleus (Sood et al., 1992), ER (Wang et al., 2006; Chanoux and Rubenstein, 2012; Pankow et al., 2015), proteasome (including ER-associated degradation pathway; Ameen et al., 2007; Chanoux and Rubenstein, 2012; Lopes-Pacheco et al., 2015; Pankow et al., 2015), Golgi apparatus (Zhang et al., 2002; Guggino and Stanton, 2006), membrane trafficking vesicles (Wang et al., 2006; Ameen et al., 2007; Chanoux and Rubenstein, 2012), plasma membrane (Sun et al., 2000b; Klein et al., 2016; Bertrand et al., 2017), and the cytoskeleton (Monterisi et al., 2012; Edelman, 2014). Below we discuss key findings from some of the large-scale approaches applied to comprehensively investigate the biological significance of PPIs in CF. These were designed to map dynamic interactomes with expectations of discovering novel proteins associated with

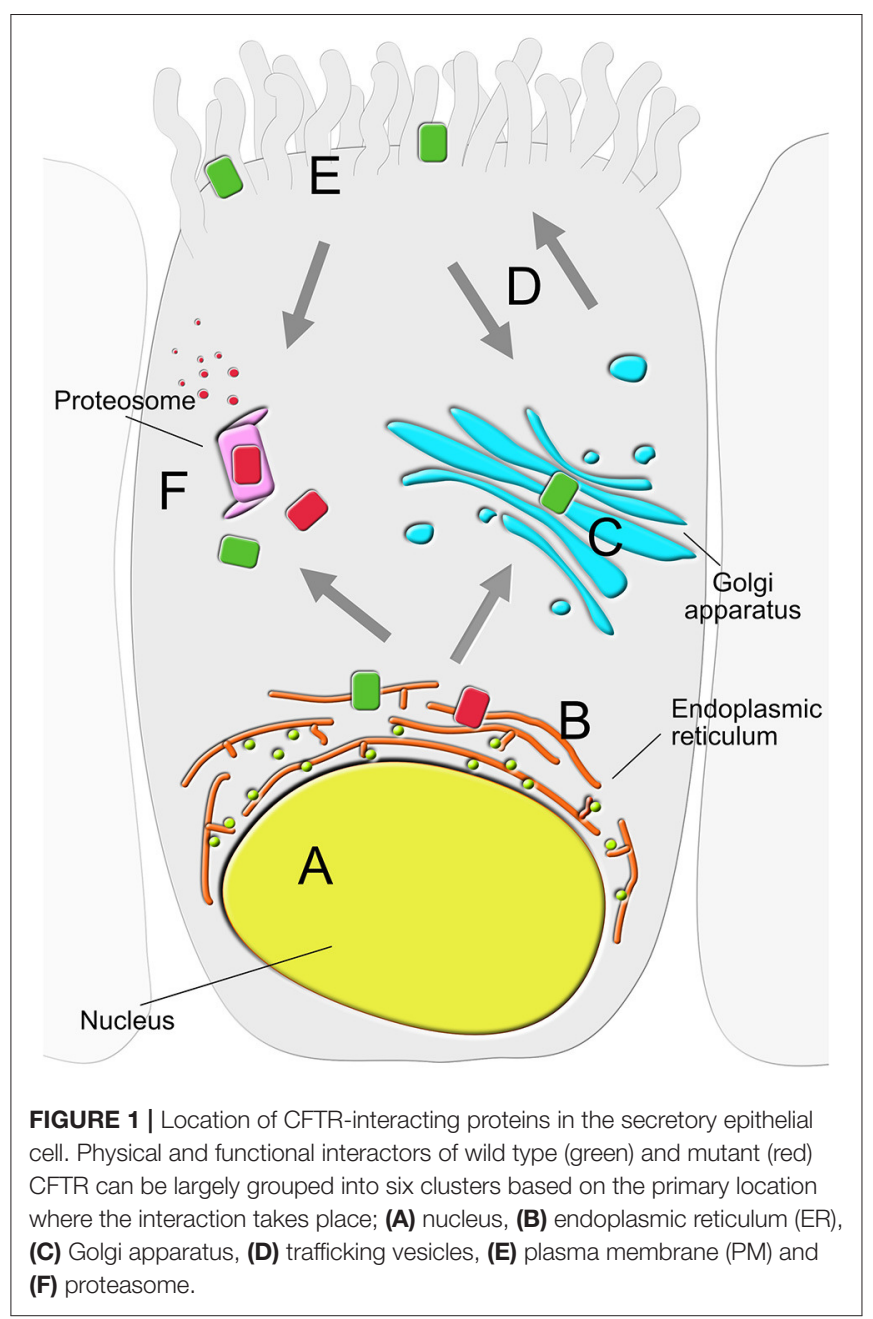


human CFTR, and to uncover new biochemical pathways that, when defective, contribute to $\mathrm{CF}$.

\section{MASS SPECTROMETRY RESULTS}

Mass spectrometry has become a popular method for characterizing the protein components of biological systems as it provides a framework for gaining insights into the composition of molecular complexes with possible regulation mechanism and function (Cravatt et al., 2007). One early report of using large-scale proteomics to understand global CFTR protein interactions came from Wang et al. (2006), in which they applied mass spectrometry through use of Multidimensional Protein-Identification Technology (MudPIT) (Wolters et al., 2001) to analyze chaperone assemblies that regulate CFTR folding and transport (Wang et al., 2006). The number of CFTR-associated proteins, including known CFTR-binding chaperones and unknown interactors, identified in this study was unprecedented. Three Hsp90 co-chaperones were found to be involved in regulating channel folding and/or export from the ER, and by using RNA-interference (RNAi) knockdown, Aha1 was discovered to inhibit coupling of F508del CFTR to the ER export machinery (Wang et al., 2006). The authors discovered that a reduced level of Aha1 alters the Hsp90CFTR interaction by promoting the transition from folding to the export pathways. This study introduced the concept of the CFTR "chaperome," a dynamic cellular chaperone folding environment that can be subjected to pharmacological intervention.

Later, the group moved on to establish comprehensive dynamic interactomes for both wild type and F508del CFTR using an improved purification technology termed Cointeracting Protein Identification Technology (CoPIT) (Pankow et al., 2015). They found 638 high-confidence interactors that further enriched the previously existing core CFTR interactome. Substantial insight into the molecular mechanisms of $\mathrm{CF}$ was obtained by analyzing the extensively remodeled F508del CFTR interactome upon rescue. Until then, there were few proteins whose known interaction with CFTR involved mutant stabilization and partial restoration of channel activity. However, in this study, Pankow et al. (2015) identified 209 proteins that differ significantly in the relative amounts recovered between the F508del and wild type CFTR cell lines, representing highly enriched wt/mutant specific interactors. In addition, the F508del CFTR-specific interactome was characterized mainly by gain of novel interaction partners, which revealed distinct differences in the biogenesis of the two CFTR proteins. Not surprisingly, recruitment of chaperones and protein degradation mediators of ER quality control network was found to be enhanced in the F508del CFTR-specific interactome. Also, PPIs involved in translation, post-translational modification, protein transport and trafficking, and endocytic recycling were altered, suggesting that large aspects of CFTR biogenesis are affected by deletion of F508 (Pankow et al., 2015). Notably, the F508del CFTR interactome dynamics under a lower temperature rescue condition resulted in reduced interactions with proteins involved in ER quality control. The authors also investigated interactome remodeling upon HDACi and validated their key interactors by performing an RNA interference screen. This work from Pankow et al. is the most exhaustive proteomics project involving human CFTR performed to date reporting that the loss of F508del CFTR function involves novel associations with protein complexes and cellular pathways that differ compared to wild type (Pankow et al., 2015).

The CoPIT method has been further utilized in the most recent work by Reilly et al. (2017) to identify a novel role of the PI3K/Akt/mTOR pathway in CF (Reilly et al., 2017). They demonstrated that inhibiting the upregulated mTOR activity in F508del CF bronchial epithelial cells resulted in increased CFTR stability and expression.

\section{TWO-DIMENSIONAL GEL ELECTROPHORESIS (2-DE) APPROACHES}

Traditional proteomics methodologies involved separation of protein samples by isoelectric point and molecular weight, with the addition of mass spectrometry analysis in more recent years. This proteomic approach was first applied to the identification of protein characteristics of CF by Pollard et al. (2005) using CF cell lines IB3-1 and its wild-type CFTR-repaired daughter cell line CFTR IB3-1/S9 (Pollard et al., 2005). They identified 194 proteins including a high abundance of chaperones, proteasome proteins, and calcium signaling and pro-inflammatory proteins associated with the NFאB pathway, serving as a foundation reference database for proteomic studies on CF cells. Involvement of keratins in transferring CFTR to the plasma membrane was identified through this method by Davezac et al. (2004). Using 2-DE and Western blot approaches, another group identified proteins involved in unfolded protein response (UPR) and cell metabolism that promote low temperature-induced F508del CFTR rescue (Gomes-Alves et al., 2009). The same group later discovered that proteome modulation associated with the UPR contributes to the rescue of F508del CFTR by RXR motif inactivation (Gomes-Alves et al., 2010).

Identification of a protein with different binding affinity to the mutant form of CFTR than to CFTR wild type was also discovered using a 2-DE method. Teng et al. (2012) identified calumenin as a new CFTR chaperone that is present in higher abundance with a complex containing G551D CFTR, which is another CF-causing missense mutation resulting in altered channel activation (Teng et al., 2012). They first resolved proteins co-immunoprecipitated with G551D CFTR by 2-DE then applied mass spectrometry to spots that were different in intensity compared with the wild type distribution. By using coimmunoprecipitation, the authors were able to determine that though the basal expression of calumenin is similar between G551D and wild type CFTR-expressing cells, the amount of calumenin bound to the complex in G551D CFTR cells is higher with increased expression of Grp78, a protein involved in the UPR.

Singh et al. (2006) applied the chemical chaperone 4Phenylbutyrate (4-PBA), an oral butyrate derivative used for 
TABLE 1 | Putative CFTR-interacting targets for restoring CFTR biogenesis and function in CF identified through proteomic approaches.

\begin{tabular}{|c|c|c|c|c|c|}
\hline Targets & Function & $\begin{array}{l}\text { Interacting } \\
\text { CFTR variant }\end{array}$ & Location & Effects on CFTR biogenesis/function & Ref. \\
\hline Aha1 & $\begin{array}{l}\text { Hsp90 cochaperone ATPase } \\
\text { regulator }\end{array}$ & F508del & ER & $\begin{array}{l}\text { Downregulates the expression and } \\
\text { maturation of F508del CFTR }\end{array}$ & Wang et al., 2006 \\
\hline PABPC1 & $\begin{array}{l}\text { RNA processing and } \\
\text { co-translational control }\end{array}$ & & Cytosol & \multirow{7}{*}{$\begin{array}{l}\text { Knockdown promotes F508del CFTR } \\
\text { maturation and enhances } \\
\text { forskolin/genistein-stimulated F508del } \\
\text { CFTR channel activity in primary CF } \\
\text { epithelia }\end{array}$} & \multirow[t]{8}{*}{$\begin{array}{l}\text { Pankow et al., } \\
2015\end{array}$} \\
\hline PTBP1 & & & Nucleus & & \\
\hline YBX1 & & & Nucleus/Cytosol & & \\
\hline TRIM21 & $\begin{array}{l}\text { E3 ubiquitin-protein ligase; } \\
\text { proteosomal degradation }\end{array}$ & & Cytosol & & \\
\hline PDIA4 & $\begin{array}{l}\text { Potential novel components of } \\
\text { ER quality control }\end{array}$ & & ER & & \\
\hline SURF4 & & & ER/Golgi & & \\
\hline PTPLAD1 & & & ER & & \\
\hline LGALS3BP & $\begin{array}{l}\text { Lectin Galactoside-Binding } \\
\text { Soluble 3-Binding Protein }\end{array}$ & & Extracellular/PM & $\begin{array}{l}\text { Knockdown results in loss of expression } \\
\text { and channel activity of F508del CFTR }\end{array}$ & \\
\hline BAG3 & $\begin{array}{l}\text { Bcl-2-associated athanogene 3; } \\
\text { co-chaperone of } \mathrm{Hsp} 70 / \mathrm{Hsc} 70\end{array}$ & & Nucleus/Cytosol & $\begin{array}{l}\text { Inhibition reduces F508del CFTR } \\
\text { aggregates }\end{array}$ & Reilly et al., 2017 \\
\hline KRT18 & $\begin{array}{l}\text { Type I intermediate filament chain } \\
\text { Keratin } 18\end{array}$ & & Cytoskeleton & $\begin{array}{l}\text { Redistribution and/or decreasing the } \\
\text { expression promotes F508del CFTR } \\
\text { delivery to the plasma membrane }\end{array}$ & $\begin{array}{l}\text { Davezac et al., } \\
2004\end{array}$ \\
\hline Psme2 & $\begin{array}{l}\text { Proteasome activator } 28 \text { subunit } \\
\text { beta (PA28 } \beta)\end{array}$ & \multirow{3}{*}{$\begin{array}{l}\text { F508del \& } \\
\text { F508del/4RK- } \\
\text { CFTR }\end{array}$} & Proteasome & \multirow{3}{*}{$\begin{array}{l}\text { Differential expression pattern observed } \\
\text { with low temperature treatment for } \\
\text { F508del CFTR rescue in BHK cells }\end{array}$} & \multirow[t]{3}{*}{$\begin{array}{l}\text { Gomes-Alves } \\
\text { et al., 2009, } 2010\end{array}$} \\
\hline Cops5 & COP9 Signalosome Subunit 5 & & Cytosol & & \\
\hline RACK1 & $\begin{array}{l}\text { Receptor For Activated C } \\
\text { Kinase } 1\end{array}$ & & ER/vesicles/PM & & \\
\hline Calumenin & $\begin{array}{l}\text { Calcium-binding protein involved } \\
\text { in protein folding and sorting }\end{array}$ & G551D & ER & $\begin{array}{l}\text { Increased level of interaction with G551D } \\
\text { CFTR during maturation/trafficking } \\
\text { compared to wild type CFTR }\end{array}$ & Teng et al., 2012 \\
\hline $\begin{array}{l}\text { GRP94 } \\
\text { (HSP90B1) }\end{array}$ & Heat Shock Protein families & F508del & ER & $\begin{array}{l}\text { Association with immature CFTR upon } \\
\text { 4-PBA modulation }\end{array}$ & $\begin{array}{l}\text { Singh et al., 2006, } \\
2008\end{array}$ \\
\hline \multicolumn{6}{|l|}{$\begin{array}{l}\text { HSP84 } \\
\text { (HSP90AB1) }\end{array}$} \\
\hline \multicolumn{6}{|l|}{$\begin{array}{l}\text { GRP78 } \\
\text { (HSPA5) }\end{array}$} \\
\hline $\begin{array}{l}\text { GRP58 } \\
\text { (PDIA4) }\end{array}$ & $\begin{array}{l}\text { Protein Disulfide Isomerase } \\
\text { Family A Member } 3\end{array}$ & & & & \\
\hline STAT1 & $\begin{array}{l}\text { Signal transducer and activator } \\
\text { of transcription } 1\end{array}$ & & Cytosol/Nucleus & $\begin{array}{l}\text { Rescues F508del CFTR function better } \\
\text { than Corr-4a treatment when } \\
\text { co-expressed }\end{array}$ & $\begin{array}{l}\text { Trzcinska-Daneluti } \\
\text { et al., } 2009\end{array}$ \\
\hline FGFR1 & $\begin{array}{l}\text { Fibroblast Growth Factor } \\
\text { Receptor } 1\end{array}$ & & ER/vesicles/PM & $\begin{array}{l}\text { Inhibition of FGFR signaling pathway } \\
\text { upregulates F508del CFTR maturation }\end{array}$ & $\begin{array}{l}\text { Trzcinska-Daneluti } \\
\text { et al., } 2015\end{array}$ \\
\hline COPB1 & $\begin{array}{l}\text { Coatomer Protein Complex } \\
\text { Subunit Beta } 1\end{array}$ & Wild type & Vesicles/Golgi & Presumed wild type CFTR traffic inhibitor & $\begin{array}{l}\text { Botelho et al., } \\
2015\end{array}$ \\
\hline OR2AG1 & $\begin{array}{l}\text { Olfactory Receptor Family } 2 \\
\text { Subfamily AG Member } 1\end{array}$ & & PM & $\begin{array}{l}\text { Presumed wild type CFTR traffic } \\
\text { enhancer }\end{array}$ & \\
\hline USP19 & Ubiquitin Specific Peptidase 19 & F508del & ER/Cytosol/PM & $\begin{array}{l}\text { Rescues F508del CFTR when } \\
\text { overexpressed }\end{array}$ & $\begin{array}{l}\text { Hassink et al., } \\
2009\end{array}$ \\
\hline $\begin{array}{l}\text { NYD- } \\
\text { SP27 } \\
\text { (PLCZ1) }\end{array}$ & Phospholipase C isoform & & Cytosol & $\begin{array}{l}\text { Suppression results in increased F508del } \\
\text { CFTR trafficking and restoration of } \\
\text { pancreatic anion secretion }\end{array}$ & Zhu et al., 2003 \\
\hline HDAC7 & Histone deacetylase 7 & & Nucleus/Cytosol & $\begin{array}{l}\text { Chemical inhibition or siRNA knockdown } \\
\text { restores F508del CFTR function }\end{array}$ & Hutt et al., 2010 \\
\hline
\end{tabular}

treatment of urea cycle disorders, for their investigation of molecular networks involved in promoting CFTR processing. Proteome profiling of bronchial epithelial cells treated with the compound revealed 85 differentially expressed proteins that became part of the first pharmaco-proteomics map of CFTR (Singh et al., 2006). Later the authors further characterized their 
findings and concluded that the interactome of CFTR channel rescued by 4-PBA contains a set of HSP70 family proteins that constitute potential therapeutic networks for targeted intervention (Singh et al., 2008).

\section{FUNCTIONAL GENOMICS APPROACHES}

While the above studies examined the interactomes and expression patterns associated with various forms of CFTR using proteomics (Collawn et al., 2010), others have used a highcontent screen based on functional assays (Trzcinska-Daneluti et al., 2009). Using a high-throughput imaging system and co-expression of proteins fused to a halide-sensitive yellow fluorescent protein (YFP), Trzcinska-Daneluti et al. (2009) identified 13 proteins whose overexpression enhanced F508del CFTR rescue in both HEK293 and BHK cells. Of those, they emphasized STAT1, finding that F508del CFTR can also be rescued by knocking down PIAS1, which is a known protein inhibitor of activated STAT1. They later applied this assay with RNAi screens to identify novel suppressors of F508del CFTR maturation and discovered that inhibition of FGFR signaling by chemical compounds leads to altered chaperone expression and robust rescue of F508del mutant (Trzcinska-Daneluti et al., 2015).

Similarly, another group used a method which involved siRNA gene knock-down coupled with high content microscopy for readouts. Botelho et al. (2015) developed a plasma membrane protein traffic assay in the CF Bronchial Epithelial (CFBE) cell line that captures traffic efficiency of CFTR protein using doubletagged reporter and high-throughput (HT) microscopy (Botelho et al., 2015). Although their method is mainly reported as a new platform for HT screening drug discovery, combination with a small-scale siRNA screen enabled the authors to identify COPB1 and OR2AG1 as novel CFTR therapeutic target genes. This platform has the potential to be applied in larger scale to identify novel CFTR traffic regulators.

Putative protein targets that have been shown to influence CFTR biogenesis and function in CF using systemic proteomic approaches are listed in Table $\mathbf{1 .}$

\section{LACK OF PROTEIN COMPLEMENTATION ASSAY}

Although both protein complementation systems and purification techniques followed by mass-spectrometry are widely used methods for large-scale mapping of PPI, with respect to proteomic profiling of CFTR, our current knowledge is mainly based on data acquired via mass spectrometry approaches. This is due to the fact that two-hybrid/protein complementation systems are limited by technical challenges associated with the high-throughput study of full-length integral membrane proteins in human cells. For example, a significant limitation of the traditional two-hybrid screening system is the host organism, yeast, which does not have the same post-translational modification machinery as mammalian cells and has different membrane composition that are not ideal for studying human receptors and transporters whose protein networks dynamically change in response to ligand binding, drug inhibition and changes in microenvironment. Also, traditional two-hybrid requires the use of only the soluble domain of membrane proteins, rather than their proper full-length form, and can consequently lead to missing or spurious interaction. These factors have limited our ability to detect PPIs between human CFTR and its interacting partners using two-hybrid screening systems on a global scale. To address these problems, new proteomics screening technologies for use in mammalian cells are being developed such as the Mammalian Membrane Two-Hybrid (MaMTH) (Petschnigg et al., 2014, 2017; Saraon et al., 2017; Yao et al., 2017). Future large-scale studies using these new protein complementation assays should therefore detect additional binary CFTR interactors that will certainly complement the current mass spectrometry datasets.

\section{CONCLUDING REMARKS}

It is evident that phenotypic manifestation of CF is intricately connected with altered PPIs that are part of the proteostasis network (Amaral and Balch, 2015). Therefore, studying the differences in cellular protein expression and interaction profiles caused by the loss of CFTR or expression of mutants using functional genomics and proteomics is important for identifying novel therapeutic intervention approaches and developing new diagnostic tools. Indeed, proteomics approaches are beginning to provide immense information on the physiological function of CFTR and the environment in which it functions. This mini review has examined some of the currently available methods employed and the work dedicated to characterizing proteins associated with CFTR. The growing CFTR interactome will serve as a powerful resource for the CF community, and will be invaluable in furthering our understanding of CFTR function and in the identification of novel CF therapeutic targets. Merging genomic, proteomic, and other functional technologies will lead to a paradigm shift in CF health care and will be a step forward in personalized management of the disease (Amaral and Balch, 2015).

\section{AUTHOR CONTRIBUTIONS}

SL wrote the bulk of the manuscript and produced the figure. EA-L was involved in the writing of the manuscript and referencing. JS was involved in the editing and critical review of the manuscript. IS designed the overall layout and critically reviewed the manuscript.

\section{ACKNOWLEDGMENTS}

We thank Dr. Laura Riley for critical reading of the manuscript. Work in the I.S. lab is supported by Cystic Fibrosis Canada (\#2847), the Canadian Cancer Society Research Institute (\#703889), Genome Canada via Ontario Genomics (\#9427 \& \#9428), Ontario Research Fund (ORF/DIG-501411 \& RE08-009), Consortium Québécois sur la Découverte du Médicament and Brain Canada. 


\section{REFERENCES}

Amaral, M. D., and Balch, W. E. (2015). Hallmarks of therapeutic management of the cystic fibrosis functional landscape. J. Cyst. Fibros. 14, 687-699. doi: 10.1016/j.jcf.2015.09.006

Ameen, N., Silvis, M., and Bradbury, N. A. (2007). Endocytic trafficking of CFTR in health and disease. J. Cyst. Fibros. 6, 1-14. doi: 10.1016/j.jcf.2006. 09.002

Barabási, A.-L., Gulbahce, N., and Loscalzo, J. (2011). Network medicine: a network-based approach to human disease. Nat. Rev. Genet. 12, 56-68. doi: $10.1038 / \mathrm{nrg} 2918$

Bertrand, C. A., Mitra, S., Mishra, S. K., Wang, X., Zhao, Y., Pilewski, J. M., et al. (2017). The CFTR trafficking mutation F508del inhibits the constitutive activity of SLC26A9. Am. J. Physiol. Lung Cell. Mol. Physiol. 312, L912-L925. doi: 10.1152/ajplung.00178.2016

Blackman, S. M., Commander, C. W., Watson, C., Arcara, K. M., Strug, L. J., Stonebraker, J. R., et al. (2013). Genetic modifiers of cystic fibrosis-related diabetes. Diabetes 62, 3627-3635. doi: 10.2337/db13-0510

Botelho, H. M., Uliyakina, I., Awatade, N. T., Proença, M. C., Tischer, C., Sirianant, L., et al. (2015). Protein traffic disorders: an effective highthroughput fluorescence microscopy pipeline for drug discovery. Sci. Rep. 5:9038. doi: 10.1038/srep09038

Boucher, R. C. (2007). Cystic fibrosis: a disease of vulnerability to airway surface dehydration. Trends Mol. Med. 13, 231-240. doi: 10.1016/j.molmed. 2007.05.001

Chanoux, R. A., and Rubenstein, R. C. (2012). Molecular chaperones as targets to circumvent the CFTR defect in cystic fibrosis. Front. Pharmacol. 3:137. doi: 10.3389/fphar.2012.00137

Cheng, S. H., Gregory, R. J., Marshall, J., Paul, S., Souza, D. W., White, G. A., et al. (1990). Defective intracellular transport and processing of CFTR is the molecular basis of most cystic fibrosis. Cell 63, 827-834. doi: 10.1016/0092-8674(90)90148-8

Cheng, J., Moyer, B. D., Milewski, M., Loffing, J., Ikeda, M., Mickle, J. E., et al. (2002). A Golgi-associated PDZ domain protein modulates cystic fibrosis transmembrane regulator plasma membrane expression. J. Biol. Chem. 277, 3520-3529. doi: 10.1074/jbc.M110177200

Collawn, J. F., Fu, L., and Bebok, Z. (2010). Targets for cystic fibrosis therapy: proteomic analysis and correction of mutant cystic fibrosis transmembrane conductance regulator. Expert Rev. Proteomics 7, 495-506. doi: 10.1586/epr.10.45

Corvol, H., Blackman, S. M., Boëlle, P.-Y., Gallins, P. J., Pace, R. G., Stonebraker, J. R., et al. (2015). Genome-wide association meta-analysis identifies five modifier loci of lung disease severity in cystic fibrosis. Nat. Commun. 6:8382. doi: $10.1038 /$ ncomms 9382

Cravatt, B. F., Simon, G. M., and Yates, J. R. (2007). The biological impact of mass-spectrometry-based proteomics. Nature 450, 991-1000. doi: 10.1038 /nature 06525

Cutting, G. R. (2010). Modifier genes in Mendelian disorders: the example of cystic fibrosis. Ann. N. Y. Acad. Sci. 1214, 57-69. doi: 10.1111/j.1749-6632. 2010.05879.x

Dang, H., Gallins, P. J., Pace, R. G., Guo, X-L., Stonebraker, J. R., Corvol, H., et al. (2016). Novel variation at chrl1p13 associated with cystic fibrosis lung disease severity. Hum. Genome Var. 3:16020. doi: 10.1038/hgv.2016.20

Davezac, N., Tondelier, D., Lipecka, J., Fanen, P., Demaugre, F., Debski, J., et al. (2004). Global proteomic approach unmasks involvement of keratins 8 and 18 in the delivery of cystic fibrosis transmembrane conductance regulator (CFTR)/ $\triangle$ F508-CFTR to the plasma membrane. Proteomics 4, 3833-3844. doi: $10.1002 /$ pmic. 200400850

De Las Rivas, J., and Fontanillo, C. (2010). Protein-protein interactions essentials: key concepts to building and analyzing interactome networks. PLoS Comput. Biol. 6:e1000807. doi: 10.1371/journal.pcbi.1000807

Denning, G. M., Anderson, M. P., Amara, J. F., Marshall, J., Smith, A. E., and Welsh, M. J. (1992). Processing of mutant cystic fibrosis transmembrane conductance regulator is temperature-sensitive. Nature 358, 761-764. doi: 10.1038/355242a0

Dorfman, R. (2012). Modifier gene studies to identify new therapeutic targets in cystic fibrosis. Curr. Pharm. Des. 18, 674-682. doi: 10.2174/138161212 799315920
Drumm, M. L., Konstan, M. W., Schluchter, M. D., Handler, A., Pace, R., Zou, F., et al. (2005). Genetic modifiers of lung disease in cystic fibrosis. N. Engl. J. Med. 353, 1443-1453. doi: 10.1056/NEJMoa051469

Duan, Y., Sun, Y., Zhang, F., Zhang, W. K., Wang, D., Wang, Y., et al. (2012). Keratin K18 increases cystic fibrosis transmembrane conductance regulator (CFTR) surface expression by binding to its C-terminal hydrophobic patch. J. Biol. Chem. 287, 40547-40559. doi: 10.1074/jbc.M112.403584

Eckford, P. D., and Bear, C. E. (2011). Targeting the regulation of CFTR channels. Biochem. J. 435, e1-e4. doi: 10.1042/BJ20110461

Edelman, A. (2014). Cytoskeleton and CFTR. Int. J. Biochem. Cell Biol. 52, 68-72. doi: 10.1016/j.biocel.2014.03.018

Emond, M. J., Louie, T., Emerson, J., Zhao, W., Mathias, R. A., Knowles, M. R., et al. (2012). Exome sequencing of extreme phenotypes identifies DCTN4 as a modifier of chronic Pseudomonas aeruginosa infection in cystic fibrosis. Nat. Genet. 44, 886-889. doi: 10.1038/ng.2344

Fajac, I., and Wainwright, C. E. (2017). New treatments targeting the basic defects in cystic fibrosis. Presse Med. 46, e165-e175. doi: 10.1016/j.lpm.2017.01.024

Gomes-Alves, P., Neves, S., Coelho, A. V., and Penque, D. (2009). Low temperature restoring effect on F508del-CFTR misprocessing: a proteomic approach. J. Proteomics 73, 218-230. doi: 10.1016/j.jprot.2009.09.001

Gomes-Alves, P., Couto, F., Pesquita, C., Coelho, A. V., and Penque, D. (2010). Rescue of F508del-CFTR by RXR motif inactivation triggers proteome modulation associated with the unfolded protein response. Biochim. Biophys. Acta 1804, 856-865. doi: 10.1016/j.bbapap.2009.12.013

Grasemann, H., and Ratjen, F. (2010). Emerging therapies for cystic fibrosis lung disease. Expert Opin. Emerg. Drugs 15, 653-659. doi: $10.1517 / 14728214.2010 .517746$

Gu, Y., Harley, I. T. W., Henderson, L. B., Aronow, B. J., Vietor, I., Huber, L. A., et al. (2009a). IFRD1 polymorphisms in cystic fibrosis with potential link to altered neutrophil function. Nature 458, 1039-1042. doi: 10.1038/nature07811.IFRD1

Gu, Y., Harley, I. T. W., Henderson, L. B., Aronow, B. J., Vietor, I., Huber, L. A., et al. (2009b). Identification of IFRD1 as a modifier gene for cystic fibrosis lung disease. Nature 458, 1039-1042. doi: 10.1038/nature07811

Guggino, W. B., and Stanton, B. A. (2006). New insights into cystic fibrosis: molecular switches that regulate CFTR. Nat. Rev. Mol. Cell Biol. 7, 426-436. doi: 10.1038/nrm1949

Guggino, W. B. (2004). The cystic fibrosis transmembrane regulator forms macromolecular complexes with PDZ domain scaffold proteins. Proc. Am. Thorac. Soc. 1, 28-32. doi: 10.1513/pats.2306011

Hassink, G. C., Zhao, B., Sompallae, R., Altun, M., Gastaldello, S., Zinin, N. V., et al. (2009). The ER-resident ubiquitin-specific protease 19 participates in the UPR and rescues ERAD substrates. EMBO Rep. 10, 755-761. doi: 10.1038/embor.2009.69

Hutt, D. M., Herman, D., Rodrigues, A. P., Noel, S., Pilewski, J. M., Matteson, J., et al. (2010). Reduced histone deacetylase 7 activity restores function to misfolded CFTR in cystic fibrosis. Nat. Chem. Biol. 6, 25-33. doi: $10.1038 /$ nchembio. 275

Kerem, E., Corey, M., Kerem, B. S., Rommens, J., Markiewicz, D., Levison, H., et al. (1990). The relation between genotype and phenotype in cystic fibrosis-analysis of the most common mutation (delta F508). N. Engl. J. Med. 323, 1517-1522. doi: 10.1056/NEJM199011293232203

Kim, J. Y., Han, W., Namkung, W., Lee, J. H., Kim, K. H., Shin, H., et al. (2004). Inhibitory regulation of cystic fibrosis transmembrane conductance regulator anion-transporting activities by shank2. J. Biol. Chem. 279, 10389-10396. doi: $10.1074 /$ jbc.M312871200

Klein, H., Abu-Arish, A., Trinh, N. T. N., Luo, Y., Wiseman, P. W., Hanrahan, J. W., et al. (2016). Investigating CFTR and KCa3.1 protein/protein interactions. PLoS ONE 11:e0153665. doi: 10.1371/journal.pone.0153665

Kotlyar, M., Pastrello, C., Pivetta, F., Lo Sardo, A., Cumbaa, C., Li, H., et al. (2014). In silico prediction of physical protein interactions and characterization of interactome orphans. Nat. Methods 12, 79-84. doi: 10.1038/nmeth.3178

Li, C., and Naren, A. P. (2005). Macromolecular complexes of cystic fibrosis transmembrane conductance regulator and its interacting partners. Pharmacol. Ther. 108, 208-223. doi: 10.1016/j.pharmthera.2005.04.004

Li, C., and Naren, A. P. (2010). CFTR chloride channel in the apical compartments: spatiotemporal coupling to its interacting partners. Integr. Biol. 2, 161-177. doi: $10.1039 / \mathrm{b} 924455 \mathrm{~g}$ 
Lopes-Pacheco, M., Boinot, C., Sabirzhanova, I., Morales, M. M., Guggino, W. B., and Cebotaru, L. (2015). Combination of correctors rescue $\Delta$ F508CFTR by reducing its association with Hsp40 and Hsp27. J. Biol. Chem. 290, 25636-25645. doi: 10.1074/jbc.M115.671925

Lukacs, G. L., and Verkman, A. S. (2012). CFTR: folding, misfolding and correcting the $\Delta$ F508 conformational defect. Trends Mol. Med. 18, 81-91. doi: 10.1016/j.molmed.2011.10.003

Monterisi, S., Favia, M., Guerra, L., Cardone, R. A., Marzulli, D., Reshkin, S. J., et al. (2012). CFTR regulation in human airway epithelial cells requires integrity of the actin cytoskeleton and compartmentalized cAMP and PKA activity. J. Cell Sci. 125, 1106-1117. doi: $10.1242 /$ jcs. 089086

Okiyoneda, T., Barrière, H., Bagdány, M., Rabeh, W. M., Du, K., Höhfeld, J., et al. (2010). Peripheral protein quality control removes unfolded CFTR from the plasma membrane. Science 329, 805-810. doi: 10.1126/science.1191542

Pankow, S., Bamberger, C., Calzolari, D., Martínez-Bartolomé, S., Lavallée-Adam, M., Balch, W. E., et al. (2015). $\triangle$ F508 CFTR interactome remodelling promotes rescue of cystic fibrosis. Nature 528, 1-18. doi: 10.1038/nature15729

Pankow, S., Bamberger, C., Calzolari, D., Bamberger, A., and Yates, J. R. (2016). Deep interactome profiling of membrane proteins by cointeracting protein identification technology. Nat. Protoc. 11, 2515-2528. doi: $10.1038 /$ nprot.2016.140

Pasyk, S., Molinski, S., Yu, W., Eckford, P. D. W., and Bear, C. E. (2017). Identification and validation of hits from high throughput screens for CFTR modulators. Curr. Pharm. Des. 18, 628-641. doi: 10.2174/138161212799315957

Petschnigg, J., Groisman, B., Kotlyar, M., Taipale, M., Zheng, Y., Kurat, C. F., et al. (2014). The mammalian-membrane two-hybrid assay (MaMTH) for probing membrane-protein interactions in human cells. Nat. Methods 11, 585-592. doi: $10.1038 /$ nmeth.2895

Petschnigg, J., Kotlyar, M., Blair, L., Jurisica, I., Stagljar, I., and Ketteler, R. (2017). Systematic identification of oncogenic EGFR interaction partners. J. Mol. Biol. 429, 280-294. doi: 10.1016/j.jmb.2016.12.006

Pollard, H. B., Ji, X., Jozwik, C., and Jacobowitz, D. M. (2005). High abundance protein profiling of cystic fibrosis lung epithelial cells. Proteomics 5, 2210-2226. doi: 10.1002/pmic.200401120

Raghuram, V., Hallows, K. R., and Foskett, J. K. (2002). Yeast two-hybrid identification and analysis of protein interactions with CFTR. Methods Mol. Med. 70, 365-381. doi: 10.1385/1-59259-187-6:365

Reilly, R., Mroz, M. S., Dempsey, E., Wynne, K., Keely, S. J., McKone, E. F., et al. (2017). Targeting the PI3K/Akt/mTOR signalling pathway in cystic fibrosis. Sci. Rep. 7, 7642. doi: 10.1038/s41598-017-06588-z

Riordan, J. R., Rommens, J. M., Kerem, B., Alon, N., Rozmahel, R., Grzelczak, Z., et al. (1989). Identification of the cystic fibrosis gene: cloning and characterization of complementary DNA. Science 245, 1066-1073. doi: $10.1126 /$ science. 2475911

Riordan, J. R. (2005). Assembly of functional Cftr chloride channels. Annu. Rev. Physiol. 67, 701-718. doi: 10.1146/annurev.physiol.67.032003.154107

Riordan, J. R. (2008). CFTR function and prospects for therapy. Annu. Rev. Biochem. 77, 701-726. doi: 10.1146/annurev.biochem.75.103004.142532

Rolland, T., Taşan, M., Charloteaux, B., Pevzner, S. J., Zhong, Q., Sahni, N., et al. (2014). A proteome-scale map of the human interactome network. Cell 159, 1212-1226. doi: 10.1016/j.cell.2014.10.050

Saraon, P., Grozavu, I., Lim, S. H., Snider, J., Yao, Z., and Stagljar, I. (2017). Detecting membrane protein-protein interactions using the mammalian membrane two-hybrid (MaMTH) assay. 9, 38-54. doi: 10.1002/cpch.15

Scroggins, B. T., Robzyk, K., Wang, D., Marcu, M. G., Tsutsumi, S., Beebe, K., et al. (2007). An acetylation site in the middle domain of Hsp90 regulates chaperone function. Mol. Cell 25, 151-159. doi: 10.1016/j.molcel.2006.12.008

Singh, O. V., Vij, N., Mogayzel, P. J., Jozwik, C., Pollard, H. B., and Zeitlin, P. L. (2006). Pharmacoproteomics of 4-phenylbutyrate-treated IB3-1 cystic fibrosis bronchial epithelial cells. J. Proteome Res. 5, 562-571. doi: 10.1021/ pr050319o

Singh, O. V., Pollard, H. B., and Zeitlin, P. L. (2008). Chemical rescue of deltaF508CFTR mimics genetic repair in cystic fibrosis bronchial epithelial cells. Mol. Cell. Proteomics 7, 1099-1110. doi: 10.1074/mcp.M700303-MCP200

Slieker, M. G., Sanders, E. A. M., Rijkers, G. T., Ruven, H. J. T., and van der Ent, C. K. (2005). Disease modifying genes in cystic fibrosis. J. Cyst. Fibros. 4, 7-13. doi: $10.1016 /$ j.jcf.2005.05.006
Snider, J., Kotlyar, M., Saraon, P., Yao, Z., Jurisica, I., and Stagljar, I. (2015). Fundamentals of protein interaction network mapping. Mol. Syst. Biol. 11, 848. doi: 10.15252/msb.20156351

Sood, R., Bear, C., Auerbach, W., Reyes, E., Jensen, T., Kartner, N., et al. (1992). Regulation of CFTR expression and function during differentiation of intestinal epithelial cells. EMBO J. 11, 2487-2494.

Stanke, F., van Barneveld, A., Hedtfeld, S., Wölfl, S., Becker, T., and Tümmler B. (2014). The CF-modifying gene EHF promotes p.Phe508del-CFTR residual function by altering protein glycosylation and trafficking in epithelial cells. Eur. J. Hum. Genet. 22, 660-666. doi: 10.1038/ejhg.2013.209

Stolzenburg, L. R., Yang, R., Kerschner, J. L., Fossum, S., Xu, M., Hoffmann, A., et al. (2017). Regulatory dynamics of 11 p13 suggest a role for EHF in modifying CF lung disease severity. Nucleic Acids Res. 45, 8773-8784. doi: $10.1093 / \mathrm{nar} / \mathrm{gkx} 482$

Sun, F., Hug, M. J., Bradbury, N. A., and Frizzell, R. A. (2000a). Protein kinase A associates with cystic fibrosis transmembrane conductance regulator via an interaction with ezrin. J. Biol. Chem. 275, 14360-14366. doi: $10.1074 / j b c .275 .19 .14360$

Sun, F., Hug, M. J., Lewarchik, C. M., Yun, C. H. C., Bradbury, N. A., and Frizzell, R. A. (2000b). E3KARP mediates the association of ezrin and protein kinase A with the cystic fibrosis transmembrane conductance regulator in airway cells. J. Biol. Chem. 275, 29539-29546. doi: 10.1074/jbc.M004961200

Sun, L., Rommens, J. M., Corvol, H., Li, W., Li, X., Chiang, T. A., et al. (2012). Multiple apical plasma membrane constituents are associated with susceptibility to meconium ileus in individuals with cystic fibrosis. Nat. Genet. 44, 562-569. doi: 10.1038/ng.2221

Teng, L., Kerbiriou, M., Taiya, M., Le Hir, S., Mignen, O., Benz, N., et al. (2012). Proteomic identification of calumenin as a G551D - CFTR associated protein. PLoS ONE 7:e40173. doi: 10.1371/journal.pone.0040173

Trzcinska-Daneluti, A. M., Ly, D., Huynh, L., Jiang, C., Fladd, C., and Rotin, D. (2009). High-content functional screen to identify proteins that correct F508del-CFTR function. Mol. Cell. Proteomics 8, 780-790. doi: $10.1074 /$ mcp.M800268-MCP200

Trzcinska-Daneluti, A. M., Chen, A., Nguyen, L., Murchie, R., Jiang, C., Moffat, J., et al. (2015). RNA Interference Screen to Identify Kinases That Suppress Rescue of $\triangle$ F508-CFTR. Mol. Cell. Proteomics 14, 1569-1583. doi: 10.1074/mcp.M114.046375

Venkatesan, K., Rual, J.-F., Vazquez, A., Stelzl, U., Lemmens, I., HirozaneKishikawa, T., et al. (2009). An empirical framework for binary interactome mapping. Nat. Methods 6, 83-90. doi: 10.1038/nmeth.1280

Vidal, M., Cusick, M. E., and Barabasi, A.-L. (2011). Interactome networks and human disease. Cell 144, 986-998. doi: 10.1016/j.cell.2011.02.016

von Mering, C., Krause, R., Snel, B., Cornell, M., Oliver, S. G., Fields, S., et al. (2002). Comparative assessment of large-scale data sets of protein-protein interactions. Nature 417, 399-403. doi: 10.1038/nature750

Wainwright, C. E., Elborn, J. S., Ramsey, B. W., Marigowda, G., Huang, X., Cipolli, M., et al. (2015). Lumacaftor-ivacaftor in patients with cystic fibrosis homozygous for Phe508del CFTR. N. Engl. J. Med. 373, 220-231. doi: 10.1056/NEJMoa1409547

Wang, S., and Li, M. (2001). Molecular studies of CFTR interacting proteins. Pflugers Arch. Eur. J. Physiol. 443(Suppl. 1), 62-64. doi: 10.1007/s004240100646

Wang, X., Venable, J., LaPointe, P., Hutt, D. M., Koulov, A. V., Coppinger, J., et al. (2006). Hsp90 cochaperone Ahal downregulation rescues misfolding of CFTR in cystic fibrosis. Cell 127, 803-815. doi: 10.1016/j.cell.2006.09.043

Wang, X., Koulov, A. V., Kellner, W. A., Riordan, J. R., and Balch, W. E. (2008). Chemical and biological folding contribute to temperature-sensitive $\Delta$ F508 CFTR trafficking. Traffic 9, 1878-1893. doi: 10.1111/j.1600-0854.2008. 00806.x

Weiler, C. A., and Drumm, M. L. (2013). Genetic influences on cystic fibrosis lung disease severity. Front Pharmacol. 4:40. doi: 10.3389/fphar.2013.00040

Wolters, D. A., Washburn, M. P., and Yates, J. R. (2001). An automated multidimensional protein identification technology for shotgun proteomics. Anal. Chem. 73, 5683-5690. doi: 10.1021/ac010617e

Wright, F. A., Strug, L. J., Doshi, V. K., Commander, C. W., Blackman, S. M., Sun, L., et al. (2011). Genome-wide association and linkage identify modifier loci of lung disease severity in cystic fibrosis at $11 \mathrm{p} 13$ and 20q13.2. Nat. Genet. 43, 539-546. doi: 10.1038/ng.838 
Yao, Z., Darowski, K., St-Denis, N., Wong, V., Offensperger, F., Villedieu, A., et al. (2017). A global analysis of the receptor tyrosine kinase-protein phosphatase interactome. Mol. Cell 65, 347-360. doi: 10.1016/j.molcel.2016.12.004

Yi, S., Pierucci-Alves, F., and Schultz, B. D. (2013). Transforming growth factor- $\beta 1$ impairs CFTR-mediated anion secretion across cultured porcine vas deferens epithelial monolayer via the p38 MAPK pathway. Am. J. Physiol. Cell Physiol. 305, C867-C876. doi: 10.1152/ajpcell.00121.2013

Zhang, H., Peters, K. W., Sun, F., Marino, C. R., Lang, J., Burgoyne, R. D., et al. (2002). Cysteine string protein interacts with and modulates the maturation of the cystic fibrosis transmembrane conductance regulator. J. Biol. Chem. 277, 28948-28958. doi: 10.1074/jbc.M111706200

Zhu, H., Zhu, J. X., Lo, P. S., Li, J., Leung, K. M., Rowlands, D. K., et al. (2003). Rescue of defective pancreatic secretion in cystic-fibrosis cells by suppression of a novel isoform of phospholipase C. Lancet 362, 2059-2065. doi: $10.1016 / 50140-6736(03) 15100-8$
Zielenski, J., and Tsui, L. C. (1995). Cystic fibrosis: genotypic and phenotypic variations. Annu. Rev. Genet. 29, 777-807. doi: 10.1146/annurev.genet. 29.1.777

Conflict of Interest Statement: The authors declare that the research was conducted in the absence of any commercial or financial relationships that could be construed as a potential conflict of interest.

Copyright $\odot 2018$ Lim, Legere, Snider and Stagljar. This is an open-access article distributed under the terms of the Creative Commons Attribution License (CC $B Y)$. The use, distribution or reproduction in other forums is permitted, provided the original author(s) or licensor are credited and that the original publication in this journal is cited, in accordance with accepted academic practice. No use, distribution or reproduction is permitted which does not comply with these terms. 